\title{
Observation of the radiative decay mode of the free neutron
}

\author{
Jeffrey S. Nico ${ }^{1}$, Maynard S. Dewey ${ }^{1}$, Thomas R. Gentile ${ }^{1}$, H. Pieter Mumm ${ }^{1}$, Alan K. Thompson ${ }^{1}$, Brian M. Fisher ${ }^{2}$, \\ Isaac Kremsky ${ }^{2}$, Fred E. Wietfeldt ${ }^{2}$, Timothy E. Chupp ${ }^{3}$, Robert L. Cooper ${ }^{3}$, Elizabeth J. Beise ${ }^{4}$, Kristin G. Kiriluk ${ }^{4}$, \\ James Byrne ${ }^{5} \&$ Kevin J. Coakley ${ }^{6}$
}

The theory of quantum electrodynamics (QED) predicts that beta decay of the neutron into a proton, electron and antineutrino should be accompanied by a continuous spectrum of soft photons. While this inner bremsstrahlung branch has been previously measured in nuclear beta and electron capture decay, it has never been observed in free neutron decay. Recently, the photon energy spectrum and branching ratio for neutron radiative decay have been calculated using two approaches: a standard QED framework ${ }^{1-3}$ and heavy baryon chiral perturbation theory ${ }^{4}$ (an effective theory of hadrons based on the symmetries of quantum chromodynamics). The QED calculation treats the nucleons as point-like, whereas the latter approach includes the effect of nucleon structure in a systematic way. Here we observe the radiative decay mode of free neutrons, measuring photons in coincidence with both the emitted electron and proton. We determined a branching ratio of $(3.13 \pm 0.34) \times 10^{-3}$ (68 per cent level of confidence) in the energy region between 15 and $340 \mathrm{keV}$, where the uncertainty is dominated by systematic effects. The value is consistent with the predictions of both theoretical approaches; the characteristic energy spectrum of the radiated photons, which differs from the uncorrelated background spectrum, is also consistent with the calculated spectrum. This result may provide opportunities for more detailed investigations of the weak interaction processes involved in neutron beta decay.

The neutron is composed of two down quarks and an up quark and is stable under the strong and electromagnetic interactions. The weak interaction, however, can convert a down quark into an up quark through the emission of a virtual $W$ gauge boson that subsequently decays into an electron and an antineutrino. In QED, the decay is also accompanied by an inner-bremsstrahlung photon in the process:

$$
\mathrm{n} \rightarrow \mathrm{p}+\mathrm{e}^{-}+\bar{v}_{e}+\gamma \text {. }
$$

QED takes into account the inner bremsstrahlung produced by the electron while the heavy baryon chiral perturbation theory approach includes the photon emission from the weak interaction vertex. Because direct emission from the weak vertex contributes less than $1 \%$ to the total intensity, both the photon energy spectrum and the polarization are dominated by the electron inner bremsstrahlung. The total intensity of inner bremsstrahlung diverges logarithmically as the photon energy E goes to zero because the spectrum displays the $1 / E$ behaviour that is characteristic of all soft photon processes. However, it has long been established that this infrared divergence is cancelled in all orders of perturbation theory by higher-order virtual photon corrections to the radiationless mode ${ }^{5-7}$.
The experimental challenge for the detection of radiative neutron decay is to distinguish the low rate of radiative decay events at observable energies from the intense photon background associated with a neutron beam. The estimated branching ratio above $15 \mathrm{keV}$ is only about $3 \times 10^{-3}$, which, when coupled with the long neutron lifetime, makes the rate of detectable photons quite small. A previous experimental study of neutron radiative decay arrived at an upper limit of $6 \times 10^{-3}$ at the $90 \%$ confidence level for the branching ratio for emission of photons with an energy between 35 and $100 \mathrm{keV}$ (ref. 8 ). The report of a more recent experiment ${ }^{9}$ was disputed ${ }^{10}$ with compelling arguments. Our experiment was mounted at the NG-6 fundamental physics end-station at the Center for Neutron Research at the National Institute of Standards and Technology. It was designed to detect the coincidence of a photon and electron followed by a delayed proton, thereby reducing the probability of recording uncorrelated background events ${ }^{11}$. A strong magnetic field transported the electrons and protons away from the photon detector, which increased the solid angle for detection and minimized correlated backgrounds. The detection method used a solenoid design and proton detection scheme previously used to measure both the neutron lifetime $e^{12,13}$ and the electron-antineutrino angular correlation coefficient ${ }^{14}$. An electrostatic mirror was used to vary the rate of detected electron-proton coincidences without changing the uncorrelated photon background rate, thus providing a signature for the detection of radiative decay and an important systematic check on possible backgrounds.

The NG-6 cold neutron beam entered beryllium-coated guides and collimation originally designed for use in a neutron time-reversal violation experiment ${ }^{15}$ and adapted for this experiment by implementing slight changes in the beam optics. The collimation used a series of ${ }^{6} \mathrm{LiF}$ apertures backed with lead to define the beam, and the vacuum components were lined with ${ }^{6} \mathrm{Li}$-glass to absorb scattered neutrons, thus significantly reducing background radiation. The beam entered parallel to the $4.6 \mathrm{~T}$ magnetic field produced by a superconducting solenoid. The detection scheme for the decay products is shown in Fig. 1. To satisfy the need for a large solid-angle photon detector that can operate in a strong magnetic field and at low temperatures, a system consisting of a scintillating crystal coupled to an avalanche photodiode was employed ${ }^{16}$. The photon was detected by a single bismuth germanate (BGO) crystal viewed by a silicon avalanche photodiode (APD). As the temperature decreases, the APD gain increases, its noise decreases ${ }^{17}$, and the BGO light output increases ${ }^{18}$; these features allowed us to obtain a low-energy detection threshold of $15 \mathrm{keV}$. The BGO crystal was mounted in an aluminium

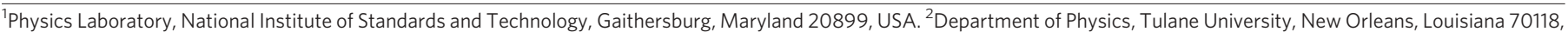

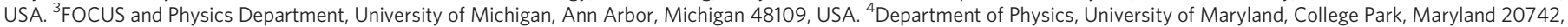

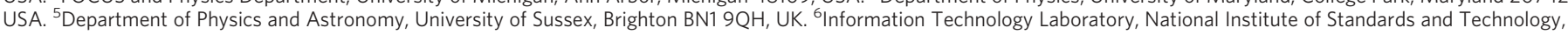
Boulder, Colorado 80305, USA. 
holder and placed below the neutron beam in the downstream end of the bore of the solenoid. The placement of the photon detector well away from the surface barrier detector (SBD) significantly reduced correlated background of external bremsstrahlung from electrons striking the SBD.

The preamplifier signal from the SBD was transmitted from high voltage via an analogue fibre optic link where it was split and amplified. Two single-channel-analyser windows were set to encompass the entire proton pulse-height peak and the electron peak just above the maximum proton peak height, so that the electron signal could not be triggered by a proton. The fast timing outputs provided the start (electron window signal) and stop (proton window signal) of a time-to-amplitude converter. If a proton stopped the time-to-amplitude converter within a $20 \mu$ s timing window, a conversion signal triggered a computer-based digital oscilloscope board that recorded the amplified electron-proton coincidence signal and the preamplifier output of the APD. Figure 2 shows a histogram of electronphoton delay times and an example of the SBD and APD waveforms.

Histograms were made of both the energy and timing spectra, and a minimal number of software cuts were placed on the data. For the proton, an energy window of twice the full width at half maximum of the gaussian energy peak was used. The entire electron spectrum above the hardware threshold of approximately $35 \mathrm{keV}$ was accepted. The electron-proton time window was 2.5 to $20 \mu$ s, where the lower limit was chosen to eliminate the influence of tails from start pulses due to events other than electrons. Studies with the beam off confirm that such events, which make up approximately $10 \%$ of the electronproton trigger rate before cuts, were typically due to beam-related $\gamma$-rays. In addition, a smaller number of events was rejected under the requirement that the start pulse should return to the baseline before accepting a stop event. Although the electron and radiative decay photon are nearly simultaneous, a window of approximately $20 \mu \mathrm{s}$ was placed on the time difference between the electron and photon to measure the uncorrelated background.

There are three main sources of correlated background that could produce a coincidence peak in the electron-photon timing spectrum: (1) external bremsstrahlung from a beta electron stopping in the $\mathrm{SBD},(2)$ cascades of $\gamma$-rays that follow neutron capture on material near the detector, and (3) high voltage discharges. Bremsstrahlung originating in the SBD is nearly indistinguishable from a radiative decay event, but the geometry of the apparatus greatly reduces the

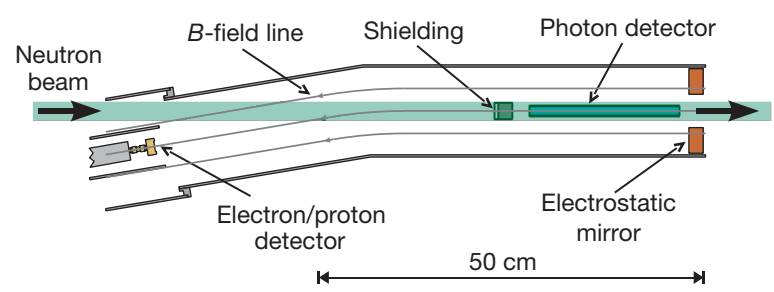

Figure 1 | Detection scheme for measuring the radiative decay of the neutron. The cold neutron beam traversed the bore of a superconducting solenoid. When a neutron decayed inside the high-field region, the charged decay products were confined to move in tight cyclotron orbits less than $1 \mathrm{~mm}$ in diameter, whose guiding centres followed the local magnetic field lines. The solenoid has a slight $\left(9.5^{\circ}\right)$ bend in the magnetic field direction at one end, allowing both the decay proton and electron to be guided out of the beam and into a silicon SBD $600 \mathrm{~mm}^{2}$ in area and $1 \mathrm{~mm}$ thick. The SBD was held at a high negative potential $(-25 \mathrm{kV})$ to accelerate the low-energy protons to detectable energy. A typical beta electron has a much higher velocity and reached the detector first, whereas the much slower proton (maximum energy of $751 \mathrm{eV}$ ) drifted to the detector a few microseconds later. The electrostatic mirror, constructed from an annulus through which the neutron beam passed, was used to reverse the direction of a proton with initial momentum directed away from the detector. Thus, the magnetic field and electrostatic mirror allowed for nearly $2 \pi$ solid-angle coverage for electron detection and up to $4 \pi$ coverage for proton detection. The photon detector and shielding lie below the neutron beam in the illustration. probability of such photons reaching the BGO crystal. Because charged particles from neutron decay are constrained to tight orbits around the magnetic field lines, they cannot strike the bore or other material in the vicinity of the photon detector. They can strike only the SBD or the downstream end of the vacuum chamber, both of which are well separated from the photon detector. Furthermore, shielding composed of ${ }^{6} \mathrm{Li}$-glass and $2 \mathrm{~cm}$ of lead partially occluded the direct line of sight between the two detectors. A simulation of the detector geometry was performed using the Monte Carlo code MCNP5 (ref. 19), and the possible contribution of external bremsstrahlung photons to the signal was determined to be $(3 \pm 3) \%$. Cascades of $\gamma$-rays that occur when a cold neutron is captured on material near the detectors can trigger both detectors. The characteristic waveforms of these events were determined by acquiring data with the accelerating voltage off, and this information was used to eliminate this source of background from the data analysis. Another possible source was high voltage discharge at the SBD. These waveforms were characterized with the beam off, and this information was also used to eliminate any significant contribution to the signal.

The experiment began in January 2004, and 23 runs with varying mirror potentials were performed from June to the end of November 2005. Some of the typical rates are given in Table 1 . The radiative decay detection rate does not change linearly with the mirror potential, in contrast with non-bremsstrahlung sources of correlated backgrounds. A Monte Carlo simulation was written to calculate the expected ratio $R_{\mathrm{ep} \gamma} / R_{\mathrm{ep}}$ as a function of mirror potential. It includes the detector geometry, four-body decay phase space of the neutron,

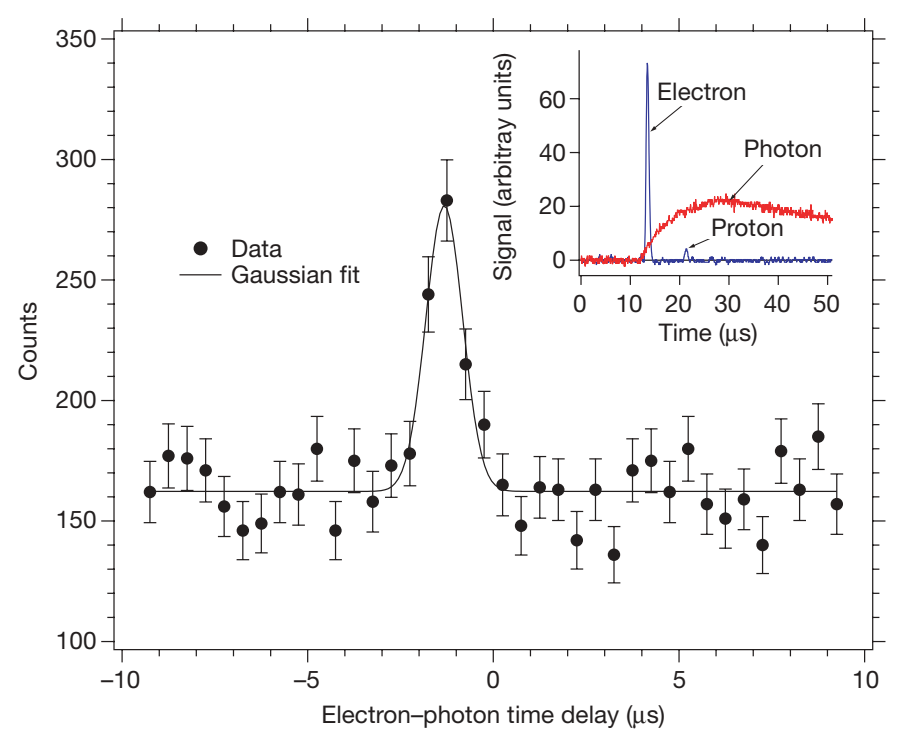

Figure 2 | Electron-photon timing spectrum for a three-day run with the mirror reflecting all protons. The spectrum shows all photons in a $20 \mu \mathrm{s}$ window for which the electron start pulse was accompanied by a delayed proton event. The error bars are statistical only and the confidence interval corresponds to $68 \%$. In the inset, the blue line shows the SBD signal for an electron event followed by a delayed proton; the red line shows the much slower pre-amplified output of the APD for a photon event occurring in coincidence with the electron. Events attributable to radiative decay photons occur as prompt events in the spectrum and appear at $-1.25 \mu$ s due to electronic delays. The approximately $1 \mu$ s width of the peak arises from the uncertainty in extracting the onset time from the comparatively slow rise time of the APD signal. The flat background rate is consistent with a calculation of the random rate of coincidences based on the rates without any coincidence requirement and the time resolution. From the electron-proton waveform, the energy of each charged particle and the difference in arrival times between the electron and proton were extracted. The particle energies were obtained by integrating the pulses, and the timing was determined from the separation of the two peaks. The same parameters were obtained from the photon waveform by fitting the preamplifier signal to an empirically determined photon pulse shape. 
Table 1 | Count rates determined from runs carried out at different mirror potentials

\begin{tabular}{lcccc}
\hline$V_{\text {mirror }}(\mathrm{V})$ & Live time $(\mathrm{d})$ & $R_{\mathrm{ep}}\left(\mathrm{s}^{-1}\right)$ & $R_{\gamma}\left(\mathrm{s}^{-1}\right)$ & $R_{\mathrm{ep} \gamma}\left(\mathrm{s}^{-1}\right)$ \\
\hline 0 & 36.1 & 5.1 & 0.020 & $(15.5 \pm 1.7) \times 10^{-5}$ \\
100 & 8.8 & 8.1 & 0.030 & $(29.2 \pm 4.5) \times 10^{-5}$ \\
200 & 8.3 & 10.8 & 0.045 & $(39.3 \pm 5.1) \times 10^{-5}$ \\
300 & 5.8 & 15.0 & 0.059 & $(77.8 \pm 7.6) \times 10^{-5}$ \\
400 & 5.6 & 17.4 & 0.068 & $(76.4 \pm 8.0) \times 10^{-5}$ \\
500 & 9.8 & 18.0 & 0.062 & $(83.2 \pm 6.3) \times 10^{-5}$ \\
700 & 2.5 & 20.1 & 0.072 & $(124 \pm 14) \times 10^{-5}$ \\
$>750$ & 11.7 & 20.2 & 0.076 & $(94.0 \pm 6.2) \times 10^{-5}$ \\
\hline
\end{tabular}

$R_{\mathrm{ep}}$ is the average rate of valid electron-proton triggers; $R_{\gamma}$ is the average rate of photon events that have a valid electron-proton trigger within a $51 \mu \mathrm{s}$ window; and $R_{\mathrm{ep} \gamma}$ is the average rate of electron-photon events with a valid proton after background subtraction (that is, the rate of events in the peak of Fig. 2). The confidence interval corresponds to $68 \%$.

and particle transport. For radiative decay events, the neutron decay kinematics and the detector acceptance yield a dependence on the mirror potential, as shown in Fig. 3. The drop in the ratio as the mirror potential goes to zero is due to the kinematics of neutron decay with photon emission. When the mirror potential is zero (or very low), only electrons and protons whose momenta have a component directed towards the SBD will be detected. Thus, the momenta of the antineutrino and emitted photon will tend to be in the opposite direction, away from the photon detector. When the mirror potential is raised above the maximum kinetic energy of the proton, the SBD detects protons emitted in all directions, and hence the photon momentum direction is less constrained.

The Monte Carlo simulation was used to extract the branching ratio, which is a single parameter that sets the scale of the ratio $R_{\mathrm{ep} \gamma} /$ $R_{\text {ep. }}$. The data in Fig. 3 were fitted to the Monte Carlo with only a single multiplicative factor, so that data taken at all mirror potentials contribute to the determination of the branching ratio. The error bars indicate statistical uncertainties only, that is, uncertainties derived from random processes. The result, with its uncertainty from the fit, is $(3.13 \pm 0.11) \times 10^{-3}$ in the energy region between 15 and $340 \mathrm{keV}$, and the $\chi^{2}$ value per degree of freedom is equal to $13.8 / 7$.

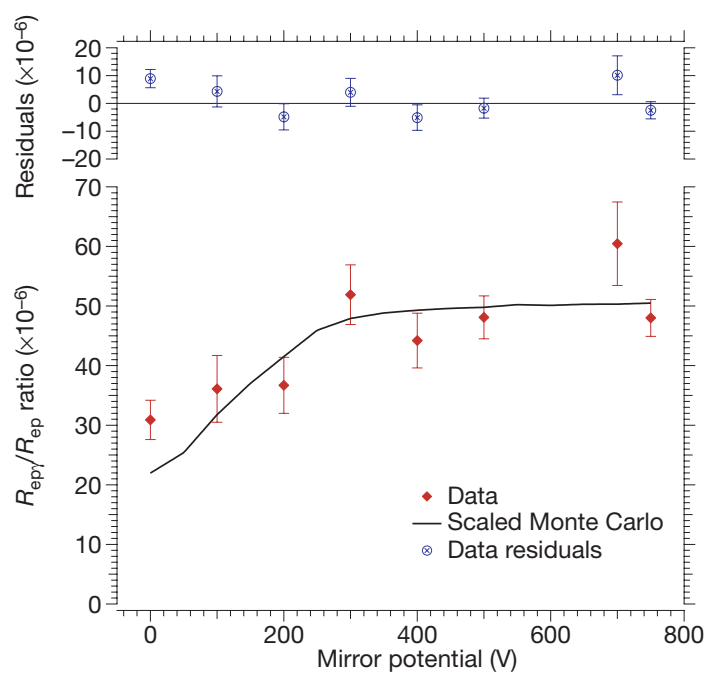

Figure 3 | Plot of the ratio $\boldsymbol{R}_{\mathrm{ep} \gamma} / \boldsymbol{R}_{\mathrm{ep}}$ versus the applied mirror potential. For each run, $R_{\mathrm{ep} \gamma}$ was obtained from the number of events in the electron-photon timing peak. This value was divided by $R_{\mathrm{ep}}$ after subtracting the background due to beam-related triggers. The runs at particular mirror potentials were repeated several times. The final value was determined by a weighted average of the individual runs. The $R_{\mathrm{ep} \gamma} / R_{\mathrm{ep}}$ ratio for the runs performed at a given potential was reproducible within statistical uncertainty, even though most runs were separated by periods of many weeks. The solid line is the result of a Monte Carlo simulation that is scaled to fit the data points so as to determine the branching ratio. The error bars indicate statistical uncertainties only. The top trace shows the residuals of the fit.
The confidence intervals quoted here and throughout are $68 \%$. The measured energy spectrum, shown in Fig. 4, is consistent with theoretical calculations ${ }^{1,4}$. No correction has been applied to these data to incorporate the photon detector response function, but modelling indicates that this correction will be small in comparison with the uncertainties of this measurement.

The dominant systematic uncertainties are associated with the gain drift and energy calibration of the photon detector and the accuracy in determining the analysis windows, which contribute a relative standard uncertainty of $7.8 \%$. Additional contributions result from the inputs of the Monte Carlo simulation, which include the spatial registration of both electric and magnetic fields relative to the detectors and the beam profile over the fiducial volume of the detector. These were varied in the Monte Carlo to yield an estimated uncertainty of 3.3\%. In addition to the correction made for external bremsstrahlung, a correction was made for the photon detector efficiency and response of $(-3.0 \pm 3.0) \%$. Other systematics include electron backscattering from the SBD, magnitude of the magnetic field, and statistical uncertainty from the Monte Carlo. Combining all the systematic corrections and uncertainties in quadrature gives a total contribution of $(0 \pm 10.5) \%$ to the branching ratio.

This measurement represents the first observation of the photons associated with the radiative decay of the neutron. The branching ratio was measured to be $(3.13 \pm 0.34) \times 10^{-3}$ and is consistent with calculations from heavy baryon chiral perturbation theory (and QED) that predict a branching ratio of $2.85 \times 10^{-3}$ in the same energy region (S. Gardner, personal communication). The total uncertainty is dominated by the contribution from systematic effects of $0.33 \times 10^{-3}$, while the statistical uncertainty contributes $0.11 \times 10^{-3}$. The photon energy spectrum is consistent with theoretical predictions, and the behaviour of the $R_{\mathrm{ep} \gamma} / R_{\mathrm{ep}}$ ratio as a function of the mirror potential coincides with the Monte Carlo prediction for radiative decay. Although the current result is limited by systematic effects, none of them presents a significant obstacle to

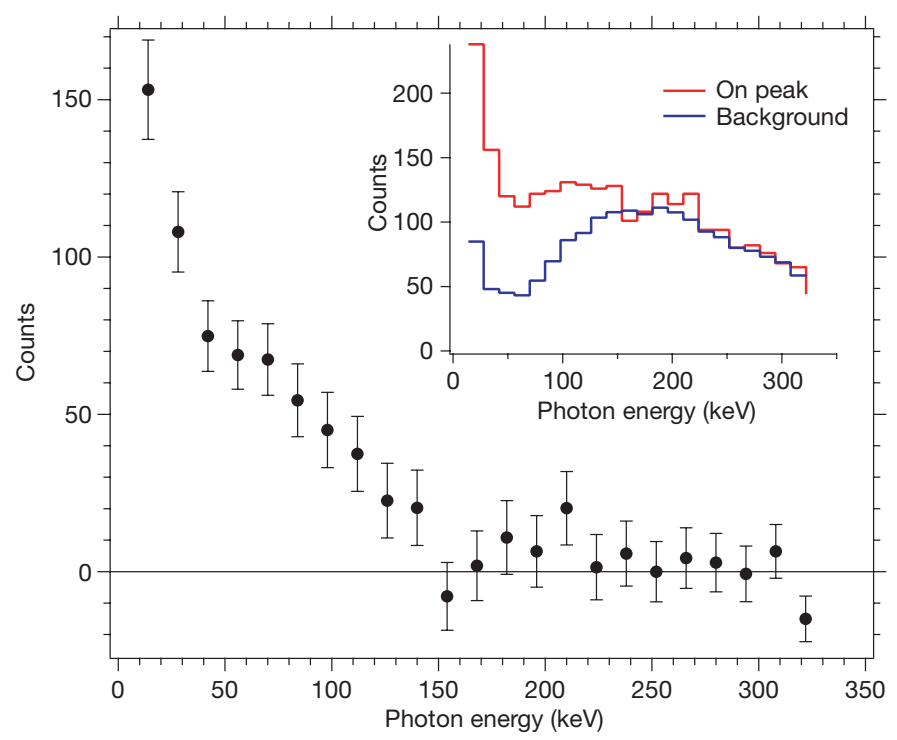

Figure 4 | Energy spectrum of photons from radiative neutron decay. The energy spectrum between 15 and $340 \mathrm{keV}$ in the electron-photon timing peak was determined by subtracting the energy spectrum of the background from that of the peak, both shown in the inset. The peak spectrum was obtained by taking a $2 \mu$ s window centred around the peak of Fig. 2, and the background spectrum was determined from an $8 \mu$ sindow in both the pre-prompt and post-prompt regions. The error bars indicate statistical uncertainties that were propagated from the difference of the two spectra. Calibrations were obtained using the $60 \mathrm{keV}$ line from ${ }^{241} \mathrm{Am}$ and the $511 \mathrm{keV}$ peak from electron-positron annihilation in the background spectrum. The spectrum shown here uses only runs in which the mirror voltage $V_{\text {mirror }}$ was greater than $750 \mathrm{~V}$ (see Table 1 ). 
improving the precision. Only a small fraction of the solid angle available for photon detection was used in this experiment. A 12element scintillation detector is under construction that should permit a precision measurement of the photon spectrum and branching ratio at a level of a few per cent. A measurement of the spectrum below the $1 \%$ level could reveal direct emission from the weak vertex. Furthermore, a measurement of the photon circular polarization could reveal information about the Dirac structure of the weak current $^{3,4}$.

\section{Received 31 August; accepted 25 October 2006.}

1. Gaponov, Yu. V. \& Khafizov, R. U. Study of radiative neutron beta decay. Phys. Atom. Nucl. 59, 1213-1220 (1996).

2. Gaponov, Yu. V. \& Khafizov, R. U. Radiative neutron $\beta$-decay and its possible experimental realization. Phys. Lett. B 379, 7-12 (1996)

3. Gaponov, Yu. V. \& Khafizov, R. U. The radiative beta-decay mode of the free neutron. Nucl. Instrum. Meth. A 440, 557-561 (2000).

4. Bernard, V., Gardner, S., Meißner, U.-G. \& Zhang, C. Radiative neutron $\beta$-decay in effective field theory. Phys. Lett. B 593, 105-114 (2004); erratum 599, 348 (2004).

5. Jauch, J. M. \& Rohrich, F. The Theory of Photons and Electrons (Addison-Wesley, Cambridge, Massachusetts, 1955).

6. Kinoshita, T. Mass singularities of Feynman amplitudes. J. Math. Phys. 3, 650-677 (1962).

7. Lee, T. D. \& Nauenberg, M. Degenerate systems and mass singularities. Phys. Rev. B 133, 1549-1562 (1964).

8. Beck, M. et al. The upper limit of the branching ratio for radiative beta decay of free neutrons. JETP Lett. 76, 332-336 (2002)

9. Khafizov, R. U. et al. Observation of the neutron radiative decay. JETP Lett. 83, 5-9 (2006).

10. Severijns, N., Zimmer, O., Wirth, H.-F. \& Rich, D. Comment on "Observation of the neutron radiative decay" by R. U. Khafizov et al. in JETP Letters 83, 5-9 (2006). JETP Lett 84, 231 (2006).
11. Fisher, B. M. et al. Detecting the radiative decay mode of the neutron. J. Res. Natl Inst. Stand. Technol. 110, 421-425 (2005).

12. Nico, J. S. et al. Measurement of the neutron lifetime by counting trapped protons in a cold neutron beam. Phys. Rev. C 71, 055502 (2005).

13. Dewey, M. S. et al. Measurement of the neutron lifetime using a proton trap. Phys. Rev. Lett. 91, 152302 (2003).

14. Byrne, J. et al. Determination of the electron-antineutrino angular correlation coefficient $\mathrm{a}_{0}$ and the parameter $|\lambda|=\left|G_{A} / G_{V}\right|$ in free neutron $\beta$-decay from measurements of the integrated energy spectrum of recoil protons stored in an ion trap. J. Phys. G 28, 1325-1349 (2002).

15. Mumm, H. P. et al. emiT: An apparatus to test time reversal invariance in polarized neutron decay. Rev. Sci. Instrum. 75, 5343-5355 (2004).

16. Gentile, T. R. et al. Particle and photon detection for a neutron radiative decay experiment. Nucl. Instrum. Meth. A (in the press).

17. Yang, L. et al. Performance of a large-area avalanche photodiode at low temperature for scintillation detection. Nucl. Instrum. Meth. A 508, 388-393 (2003).

18. Piltingsrud, H. V. The low-temperature scintillation properties of bismuth germanate and its application to high-energy gamma radiation imaging devices. J. Nucl. Med. 20, 1279-1285 (1979).

19. Brown, F. B. et al. A General Monte Carlo N-Particle Transport Code. Version 5, LANL publication LA-UR-03-1987 (Los Alamos National Laboratory, 2003).

Acknowledgements We thank S. Gardner of the University of Kentucky for discussions and her interest in this work. We acknowledge the support of the National Institute of Standards and Technology, US Department of Commerce, which provided the neutron facilities used in this work. This research was made possible in part by support from the National Science Foundation and a US Department of Energy interagency agreement.

Author Information Reprints and permissions information is available at www.nature.com/reprints. The authors declare no competing financial interests. Correspondence and requests for materials should be addressed to J.S.N. (jnico@nist.gov). 